\title{
293 cells express both epithelial as well as mesenchymal cell adhesion molecules
}

\author{
MASAKAZU INADA, GENYA IZAWA, WAKAKO KOBAYASHI and MASAYUKI OZAWA \\ Department of Biochemistry and Molecular Biology, Graduate School of Medical and \\ Dental Sciences, Kagoshima University, Kagoshima 890-8544, Japan
}

Received July 17, 2015; Accepted February 24, 2016

DOI: $10.3892 / \mathrm{ijmm} .2016 .2568$

\begin{abstract}
The 293 cell line, used extensively in various types of studies due to the ease with which these cells can be transfected, was thought to be derived by the transformation of primary cultures of human embryonic kidney cells with sheared adenovirus type 5 DNA. Although the 293 cells were assumed to originate from epithelial cells, the exact origin of these cells remains unknown. Previous attempts to characterize these cells combined immunostaining, immunoblot analysis and microarray analysis to demonstrate that 293 cells express neurofilament subunits, $\alpha$-internexin, and several other proteins typically found in neurons. These findings raised the possibility that the 293 cell line may have originated from human neuronal lineage cells. Contrary to this suggestion, in this study, we found that the 293 cells expressed $\mathrm{N}$-cadherin and vimentin, which are marker proteins expressed in mesenchymal cells. Furthermore, the 293 cells also expressed E-cadherin, cytokeratins 5/8 and desmoglein 2, which are epithelial cell markers. When the cells, primarily cultured from the kidneys of Clawn miniature swine and passaged 10-15 generations [termed porcine kidney epithelial (PKE) cells] were examined, they were found to be positive for the expression of both mesenchymal and epithelial markers. Thus, transformation by adenovirus was not necessary for the cells to express $\mathrm{N}$-cadherin. Occludin and zonula occludens (ZO)-1, two components of tight junctions in epithelial and endothelial cells, were detected in the 293 and the PKE cells. Thus, the findings of the present study demonstrate that 293 cells retain several characteristics of epithelial cells.
\end{abstract}

Correspondence to: Professor Masayuki Ozawa, Department of Biochemistry and Molecular Biology, Graduate School of Medical and Dental Sciences, Kagoshima University, 8-35-1 Sakuragaoka, Kagoshima 890-8544, Japan

E-mail:mozawa@m.kufm.kagoshima-u.ac.jp

Abbreviations: Ad5, adenovirus 5; E1, early region 1; MDCK, Madin-Darby canine kidney; NF, neurofilament; PBS, phosphatebuffered saline; PKE, porcine kidney epithelial

Key words: cadherin, cytokeratin, desmoglein, desmosome, vimentin

\section{Introduction}

The 293 cells were thought to be derived from primary human embryonic kidney cells by transformation with sheared fragments of adenovirus 5 (Ad5) DNA and contain nucleotides 1-4344 of Ad5, comprising the early region 1 (E1) transforming sequences integrated into chromosome $19(1,2)$. Since the isolation of these cells over 30 years ago, the 293 cell line has been widely used for the production of E1-deleted Ad vectors and in a number of transfection studies (3).

Although 293 cells express cytokeratins, which are epithelial cell markers, a previous study used a combination of immunostaining, immunoblot analysis and microarray analysis to demonstrate that 293 cells express neurofilament (NF) subunits, $\alpha$-internexin, and several other proteins typically found in neurons. These findings raised the possibility that the 293 cell line was derived from human neuronal lineage cells transformed by adenovirus (4). Although the presence of keratin proteins is characteristic of the early stage differentiation of epithelial cells and is unusual in neurons of the human or the rodent central nervous systems, these proteins are found in transformed lines of undoubted neuronal origin. For example, the rat PC12 line, derived from the adrenal gland, and NTera-2 cells derived from human embryonal carcinoma cells, express keratins, all three NF triplet protein subunits and vimentin (5-6). Human tumors, which contain NFs and keratins, have also been described (8).

Despite extensive analysis of neural cell marker expression in 293 cells, the expression of epithelial cell markers other than cytokeratins has not yet been studied, to the best of our knowledge. Herein, we examined the expression of E-cadherin, zonula occludens (ZO)-1, occludin and desmoglein 2, which are proteins involved in the formation and maintenance of cell-cell junctions (9-20). These proteins are components of the specialized junctional structure, consisting of the tight junction (occludin and ZO-1), the adherens junction (E-cadherin), and the desmosome (desmoglein 2). These structures are found at the apical area of lateral cell-cell contacts (9). Tight junctions are located in the most apical contact region, which constitutes the transepithelial permeability barrier. This unique junction is formed by the association of claudins and occludin (two transmembrane components of tight junctions $(10,11)$ with $\mathrm{ZO}-1$ and other cytoskeletal proteins $(12,13)$. The adherens junction is located proximal to the tight junction. The main adhesion receptors 
within the adherens junctions are the classic cadherins (14), the cytoplasmic domain of which is associated with $\beta$-catenin $(15,16)$. $\beta$-catenin, in turn, associates with $\alpha$-catenin to generate a three protein complex $(17,18)$. Desmosomes are multi-unit, protein hetero-complexes that contain desmocollin and desmoglein, two glycoproteins of the cadherin family $(19,20)$. They are located basal to the adherens junction and are associated with intermediate filaments. In simple epithelia, these three junction structures are typically aligned in the order described above, although desmosomes are also independently distributed throughout other areas of the cell membrane $(19,20)$.

In the present study, we found that the 293 cells expressed $\mathrm{N}$-cadherin, a cell adhesion protein expressed in neural cells However, the 293 cells also expressed cytokeratins 5/8, as well as desmoglein 2, which are epithelial cell markers. Low expression levels of E-cadherin were detected in the 293 cells using immunoblot analysis, but not by immunostaining. The cells primarily cultured from the kidneys of Clawn miniature swine and passaged 10-15 generations [termed porcine kidney epithelial (PKE) cells], tested positive for the expression of cytokeratins and $\mathrm{N}$-cadherin. Thus, transformation by adenovirus was not necessary for the cells to express $\mathrm{N}$-cadherin. Occludin, an integral component of tight junctions in epithelial and endothelial cells, was detected in both the 293 and the PKE cells. Thus, the findings of the present study demonstrate that 293 cells retain several characteristics of epithelial cells.

\section{Materials and methods}

Cells and transfection. The 293 cells were provided by Dr Tatsuhiko Furukawa (Department of Molecular Oncology, Kagoshima University, Kagoshima, Japan). The cells were cultured in Dulbecco's modified Eagle medium (DMEM) supplemented with $10 \%$ fetal calf serum. DLD1, a human colorectal adenocarcinoma cell line, was provided by Dr Shintaro T. Suzuki (Kwansei Gakuin University, Kobe, Japan). The PKE cells, provided by Dr Takami Matsuyama (Department of Immunology, Kagoshima University), were cells primarily cultured from the kidneys of Clawn miniature swine [a swine strain established by Japanese scientists (21)] and passaged 10-15 generations. Madin-Darby canine kidney (MDCK) cells were provided by Dr Yasushi Daikuhara (Kagoshima University Dental School, Kagoshima, Japan). The expression vector encoding hemagglutinin (HA)-tagged fulllength E-cadherin was prepared as previously described (22). The vector contains neo gene, which confers G418-resistance. As a control, an empty vector without E-cadherin gene was used yielding $\mathrm{nH}-2$ and $\mathrm{nH}-6$ clones. Transfection of 293 cells with the HA-tagged E-cadherin vector resulted in EH-5 and EH-13 clones. The cells $\left(5 \times 10^{5}\right)$ were transfected with the expression vector $(10 \mu \mathrm{g})$ using the calcium-phosphate transfection method as previously described (15), and stably transfected cells were selected in G418-containing medium. Isolated G418-resistant clones were tested for the expression of the transfected construct by immunofluorescence microscopy and immunoblot analysis.

Antibodies and reagents. Mouse monoclonal antibodies (mAbs) against E-cadherin (catalogue no. 610182), $\beta$-catenin (catalogue no. 610153), fibronectin (catalogue no. 610077) and plakoglobin ( $\gamma$-catenin; catalogue no. 610253) were obtained from BD Transduction Laboratories (Lexington, KY, USA). Pan-cadherin mAb (catalogue no. C1821-100UL) was obtained from Sigma-Aldrich (St. Louis, MO, USA). Rabbit polyclonal anti-occludin (catalogue no. 71-1500), anti-ZO-1 (catalogue no. 61-7300) and mouse monoclonal anti-vimentin (catalogue no. 18-0052) antibodies were purchased from Zymed Laboratories (South San Francisco, CA, USA). A mouse mAb specific for cytokeratins 5/8 (catalogue no. MAB3228) was acquired from Merk Millipore Ltd. (Tokyo, Japan). A mouse $\mathrm{mAb}$ against desmoglein 2 was obtained from Progen Biotechnik GmbH (Heidelberg, Germany). A rat mAb (3F10; catalogue no. 11867423001) directed against HA was purchased from Roche Applied Science (Mannheim, Germany). All secondary antibodies (fluorescein-, rhodamineand peroxidase-conjugated) were obtained from Jackson ImmunoResearch Laboratories, Inc. (West Grove, PA, USA).

Immunoblot analysis. Immunoblot analysis was performed essentially as previously described (22). Briefly, the cells were lysed by boiling in SDS sample buffer for $5 \mathrm{~min}$. Proteins (30-50 $\mu \mathrm{g}$ ) were separated by SDS-PAGEand then transferred onto nitrocellulose membranes (Schleicher \& Schuell, Keene, NH, USA). The membranes were incubated with the appropriate primary antibodies diluted at 1:1,000, followed by incubation in horseradish peroxidase-conjugated secondary anti-mouse (catalogue no. 315-036-045) or anti-rabbit (catalogue no. 111-036-045) antibodies (Jackson ImmunoResearch Laboratories, Inc.) diluted at 1:1,000. Proteins were visualized using the enhanced chemiluminescence (ECL) system (Amersham Pharmacia Biotech, Piscataway, NJ, USA).

Immunofluorescence staining. Immunofluorescence staining of the cells was performed as previously described (23) with some modifications. The cells were cultured on coverslips for $48 \mathrm{~h}$ prior to fixation. The cells were then fixed with $3 \%$ paraformaldehyde in phosphate-buffered saline (PBS) for $20 \mathrm{~min}$ at room temperature. Following 3 washes in PBS containing $50 \mathrm{mM}$ $\mathrm{NH}_{4} \mathrm{Cl}$, the cells were permeabilized using $0.1 \%$ Triton $\mathrm{X}-100$ in PBS for $5 \mathrm{~min}$. After washing in PBS, the cells were soaked in blocking solution (PBS containing 5\% fetal calf serum) for $15 \mathrm{~min}$, and then incubated for $30 \mathrm{~min}$ with primary antibodies diluted in blocking solution. After washing 3 times in PBS, the cells were incubated with rhodamine- or fluorescein-conjugated secondary antibodies. The cells were analyzed as previously described (24) using an Olympus microscope.

\section{Results}

Compared with MDCK cells, PKE and 293 cells express reduced amounts of E-cadherin. E-cadherin is a major cell adhesion molecule in epithelial cells and an epithelial cell marker (25). Although the immunofluorescence staining of MDCK cells, a typical epithelial cell line, with E-cadherin antibodies revealed strong membrane staining, the PKE and the 293 cells exhibited no membrane staining (Fig. 1A, upper panels). In a control experiment, we noted clear membrane staining for $\beta$-catenin, a cytoplasmic subunit of the transmembrane cell adhesion cadherin complex, in these 3 cell lines (Fig. 1A, lower panels).

The immunoblot detection of E-cadherin in these cells revealed that E-cadherin expression in the PKE and the 

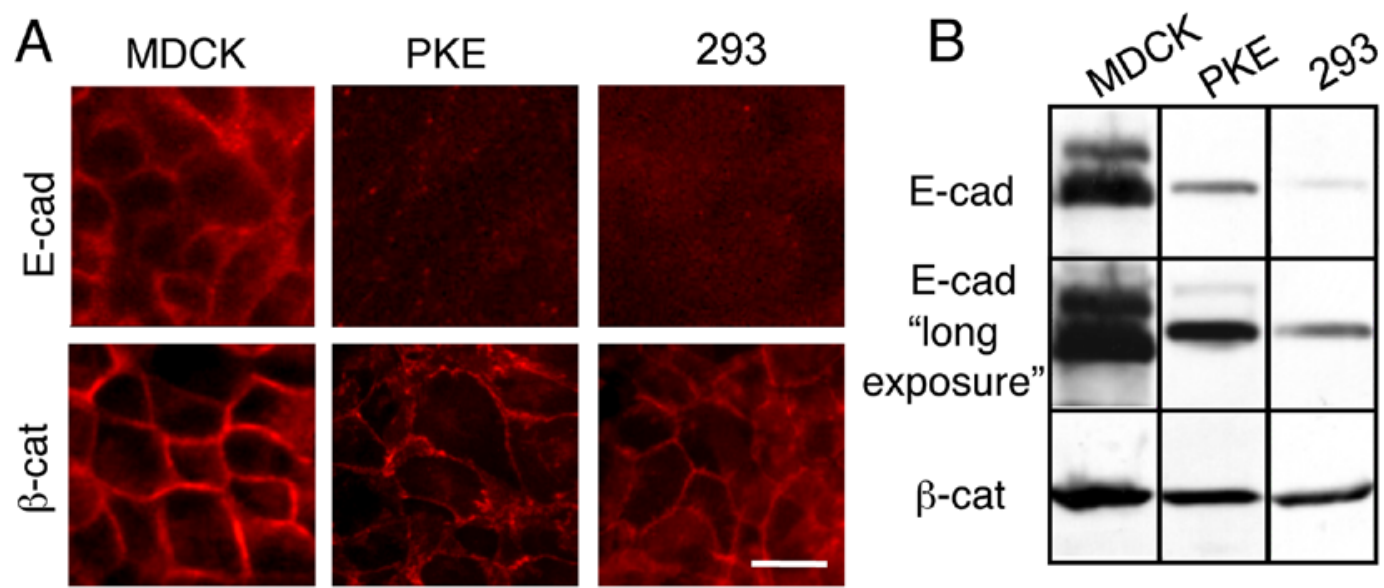

Figure 1. Detection of E-cadherin (E-cad) and $\beta$-catenin ( $\beta$-cat) in Madin-Darby canine kidney (MDCK), porcine kidney epithelial (PKE) and 293 cells. (A) MDCK, PKE and 293 cells were cultured on coverslips for $24 \mathrm{~h}$ and then examined for E-cad or $\beta$-cat using immunofluorescence microscopy. Scale bar, $25 \mu \mathrm{m}$. (B) Cells were directly lysed in SDS-sample buffer, subjected to SDS-PAGE, and examined by immunoblot analysis with E-cad and $\beta$-cat antibodies. The lower panel of E-cad shows a longer exposure time (by a factor of five) as compared with the upper panel, to allow the detection of small amounts of E-cad in PEK and 293 cells.

A

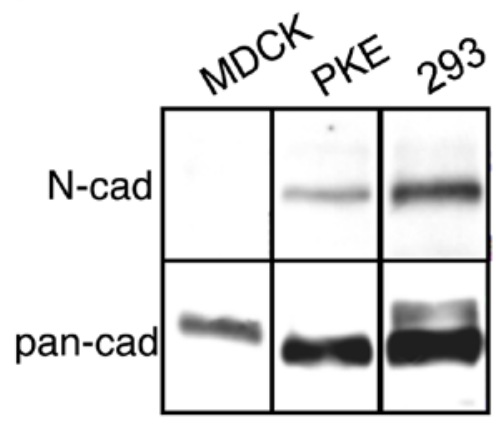

B MDCK
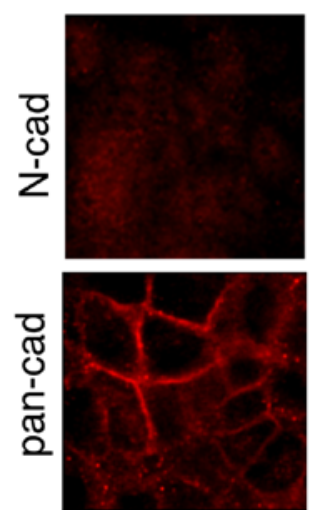

PKE
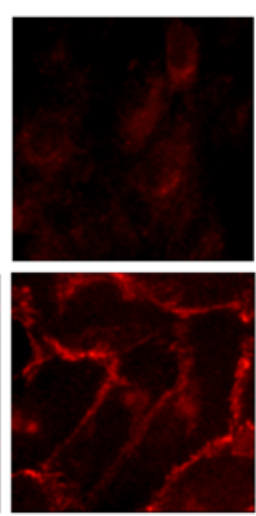

293
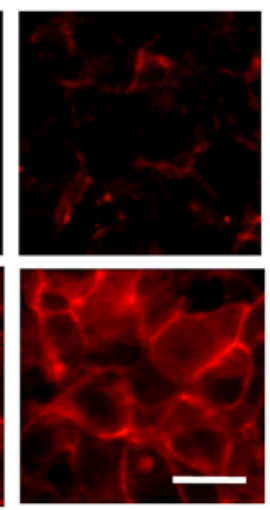

Figure 2. Detection of N-cadherin (N-cad) and pan-cadherin (pan-cad) in Madin-Darby canine kidney (MDCK), porcine kidney epithelial (PKE) and 293 cells. (A) Cells were directly lysed in SDS-sample buffer, subjected to SDS-PAGE, and examined by immunoblot analysis with N-cad or pan-cad antibodies. (B) Cells were cultured on coverslips for $24 \mathrm{~h}$ and then examined for $\mathrm{N}$-cad and pan-cad antibodies using immunofluorescence microscopy. Scale bar, $25 \mu \mathrm{m}$.

293 cells was markedly decreased compared with its expression level in the MDCK cells (Fig. 1B).

293 and PKE cells express $N$-cadherin. The immunoblot detection of N-cadherin in the PKE and the 293 cells revealed high expression levels of N-cadherin (Fig. 2A). The MDCK cells, on the other hand, did not express $\mathrm{N}$-cadherin (Fig. 2A), which was in agreement with previous findings (26). Immunoblot analysis using pan-cadherin antibodies raised against a synthetic peptide corresponding to the C-terminal amino acids of chicken N-cadherin, revealed that the PKE and the 293 cells expressed a protein with the same electrophoretic mobility as N-cadherin (Fig. 2A). Although the MDCK cells do not express $\mathrm{N}$-cadherin, they express a protein that migrates slowly relative to $\mathrm{N}$-cadherin. This protein has been shown to be K-cadherin (23,27). The 293 cells also expressed a protein with a slightly slower electrophoretic mobility (Fig. 2A). Although this protein has not been identified definitively, we conjecture that this protein is the precursor form of $\mathrm{N}$-cadherin, containing the prosequence.
The immunofluorescence staining of the cells with pancadherin antibodies revealed clear membrane staining (Fig. 2B). Thus, the proteins recognized by the pan-cadherin antibodies were present on the membrane. No heterogeneity of expression within the cell lines was noted. These results are consistent with a previous observation that $\mathrm{N}$-cadherin is expressed endogenously at cell-cell contact sites in 293 cells (28).

293, PKE and MDCK cells express cytokeratins 5/8. The MDCK cells exhibited strong and marked cytoplasmic and filamentous staining with a pan-keratin antibody mix containing $\mathrm{mAb}$ specific for keratin 5 and $\mathrm{mAb}$ specific for keratin 8 (Fig. 3A, upper panels). This staining pattern was also observed in the PKE and the 293 cells (Fig. 3A, upper panels). No heterogeneity of expression within the cell lines was noted as expected for typical kidney-derived cell lines and the 293 cells.

293, PKE and MDCK cells express ZO-1 and occludin. Although the decreased expression of E-cadherin and the unexpected expression of $\mathrm{N}$-cadherin argue against the 

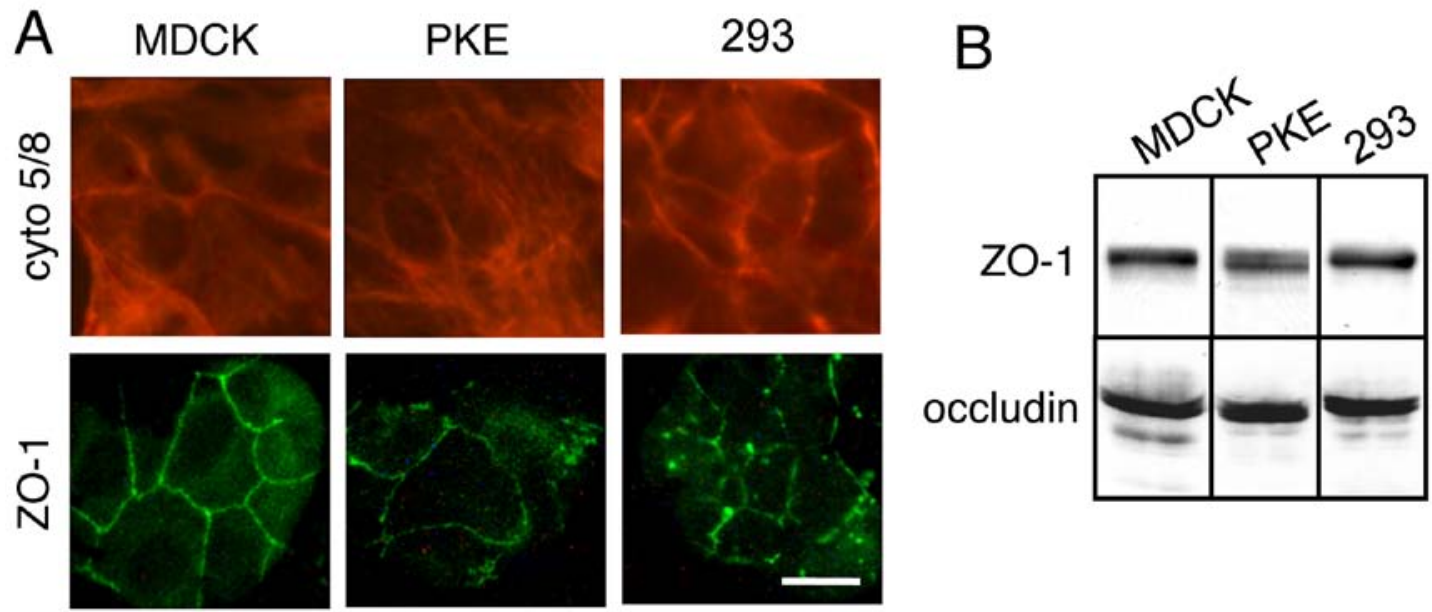

Figure 3. Detection of cytokeratin 5/8 (cyto 5/8), zonula occludens (ZO)-1, and occludin in Madin-Darby canine kidney (MDCK), porcine kidney epithelial (PKE) and 293 cells. (A) Cells were cultured on coverslips for $24 \mathrm{~h}$ and then examined for cyto $5 / 8$ or ZO-1 using immunofluorescence microscopy. Scale bar, $25 \mu \mathrm{m}$. (B) Cells were directly lysed in SDS-sample buffer, subjected to SDS-PAGE, and examined by immunoblotting with ZO-1 and occludin antibodies.
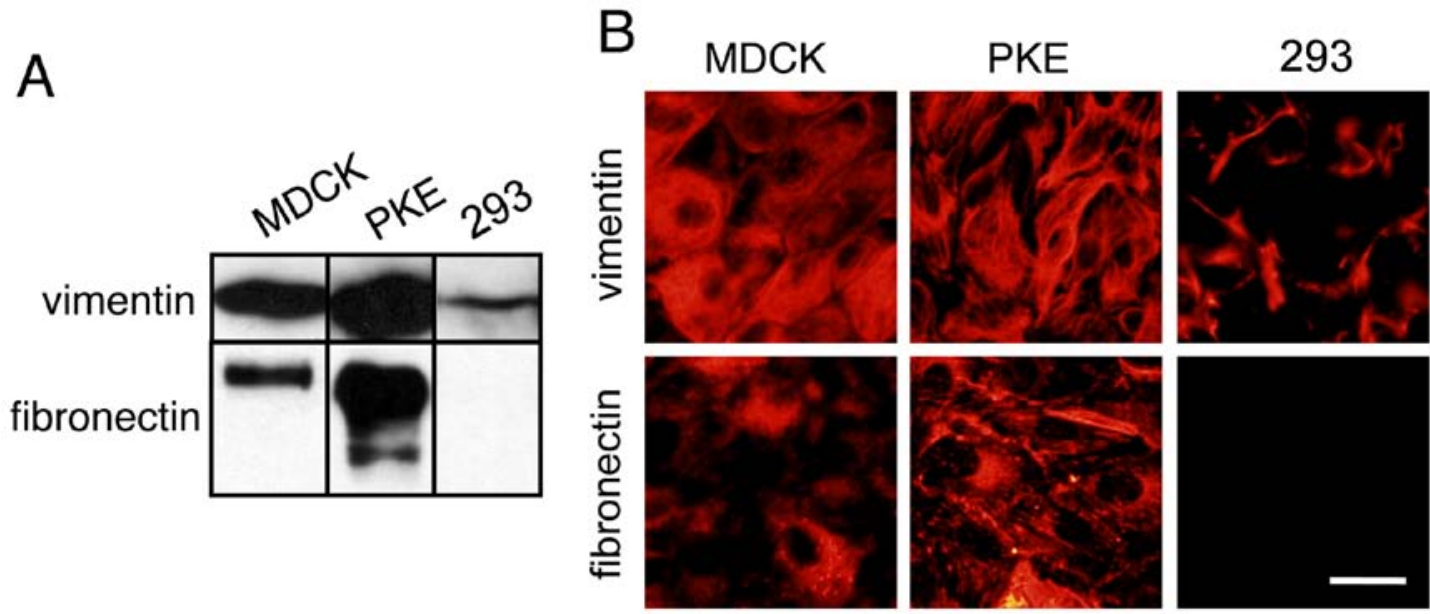

Figure 4. Detection of vimentin and fibronectin in Madin-Darby canine kidney (MDCK), porcine kidney epithelial (PKE) and 293 cells. (A) Cells were directly lysed in SDS-sample buffer, subjected to SDS-PAGE, and examined by immunoblot analysis with vimentin or fibronectin antibodies. (B) Cells were cultured on coverslips for $24 \mathrm{~h}$ and then examined for vimentin or fibronectin antibodies by immunofluorescence microscopy. Scale bar, $25 \mu \mathrm{m}$.

epithelial cell origin of 293 cells, the expression of keratin 5/8 supports the notion that they are epithelial cells. To examine the epithelial nature of 293 cells, we determined the expression levels of ZO-1 and occludin in these cells. These proteins are components of tight junctions in epithelial and endothelial cells (11). Immunoblot analysis of the cells revealed that the PKE and 293 cells, as well as the MDCK cells, expressed ZO-1 and occludin (Fig. 3B).

Immunofluorescence staining of these cells with $\mathrm{ZO}-1$ antibodies revealed strong membrane staining (Fig. 3A, lower panels). Thus, transport of the tight junction component, ZO-1, from its site of biosynthesis occurred. No heterogeneity of expression within the cell lines was noted. Immunostaining for occludin revealed similar membrane staining patterns (data not shown).

MDCK and PKE cells express the mesenchymal markers, vimentin and fibronectin. The immunoblot detection of vimentin in the MDCK and the PKE cells revealed high expression levels of vimentin (Fig. 4A). The 293 cells, on the other hand, expressed vimentin at a reduced level (Fig. 4A). Immunoblot analysis of fibronectin revealed that the PKE cells expressed a large amount of fibronectin (Fig. 4A). Although the MDCK cells expressed a small amount of fibronectin, the 293 cells did not express detectable amounts of fibronectin.

Consistent with the results of the immunoblot analysis, immunofluorescence staining of the cells with vimentin and fibronectin antibodies revealed that the PKE cells expressed these proteins (Fig. 4B). The 293 cells expressed vimentin at a low level.

Although desmoglein 2 is expressed in the 293 cells, it remains localized in intracellular compartments. The immunoblot detection of desmoglein 2 in the 293 cells revealed high expression levels (Fig. 5A). Immunofluorescence staining of the 293 cells, however, revealed no membrane staining, although the staining of the DLD1 cells revealed clear membrane staining (Fig. 5B). Thus, desmoglein 2 remained localized in intracellular compartments. No heterogeneity of expression within the cell lines was noted. 

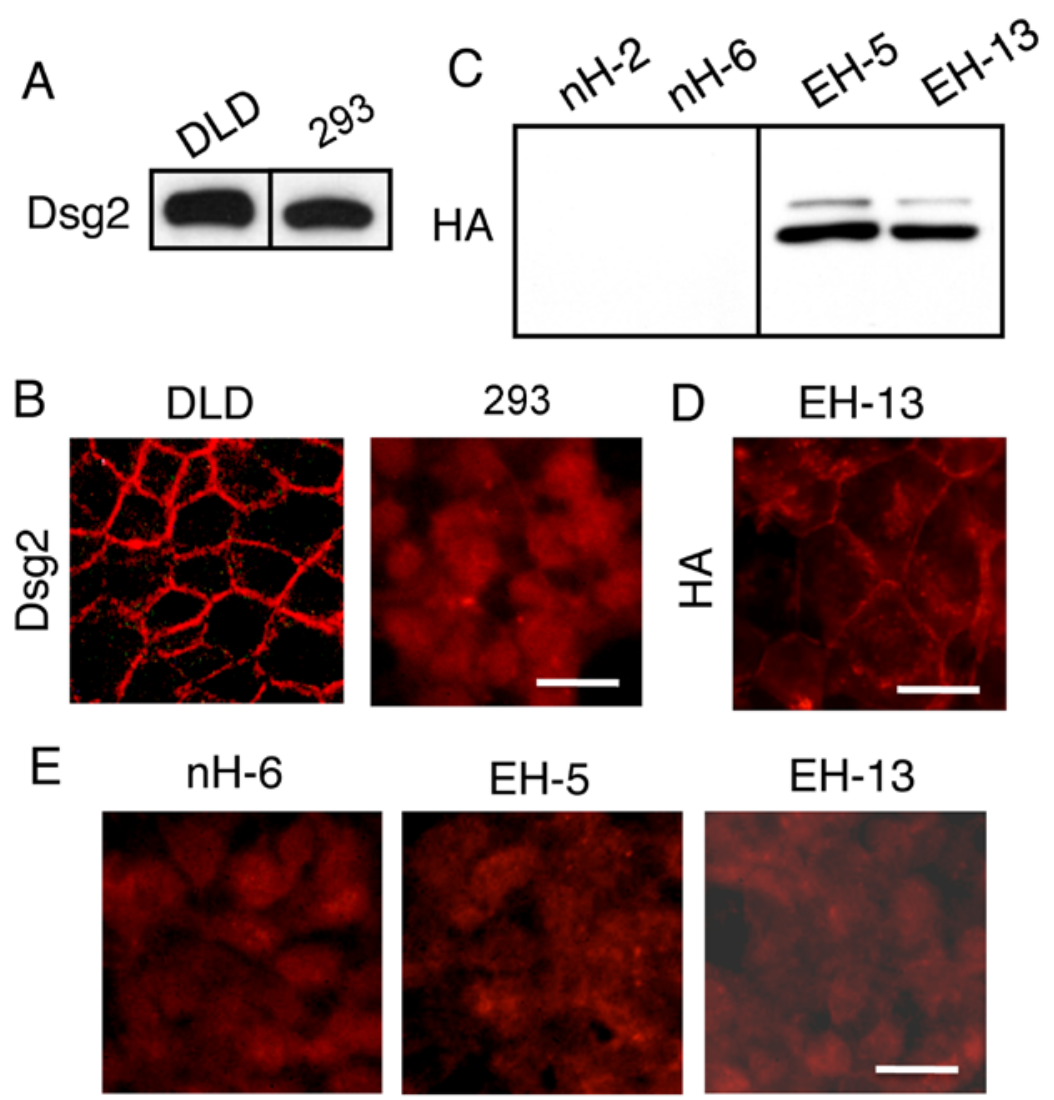

Figure 5. Detection of desmoglein 2 (Dsg2) in 293 cells. (A) DLD1 (colon cancer cell line) or 293 cells were directly lysed in SDS-sample buffer, subjected to SDS-PAGE, and examined by immunoblotting with Dsg2 antibodies. (B) Cells (DLD1 and 293) were cultured on coverslips for $24 \mathrm{~h}$ and then examined by immunofluorescence microscopy with Dsg2 antibodies. (C) 293 cells were transfected with an hemagglutinin (HA)-tagged E-cadherin expression vector. 293 cell clones that stably expressed HA-tagged E-cadherin (EH-5 and EH-13), and clones that were negative for E-cadherin (nH-2 and nH-6) were isolated. These clones were subjected to (C) immunoblot analysis or (D) immunofluorescence staining with anti-HA antibodies. (E) These cells were cultured on coverslips for $24 \mathrm{~h}$ and then examined with Dsg2 antibodies by immunofluorescence microscopy. Scale bars, $25 \mu \mathrm{m}$.

These observations suggested that low E-cadherin expression levels were responsible for the failure of desmosome assembly. In an attempt to restore the localization of desmosomes in the membrane through increased E-cadherin expression, the 293 cells were transfected with the expression vector for HA-tagged E-cadherin and stable transfectants were isolated following selection with G418 (Fig. 5C). In these cells, E-cadherin was detected mainly on the surface membrane as revealed by staining with anti-HA antibodies (Fig. 5D). Immunofluorescence staining of these E-cadherin-expressing cells with desmoglein 2 antibodies revealed no membrane staining (Fig. 5E). Thus, E-cadherin expression in 293 cells is insufficient for the cell surface localization of desmoglein 2.

\section{Discussion}

A thorough analysis of the 293 cells, thought to have been derived from human embryonic kidney cells that had been transformed by adenovirus 5 (Ad5), notably revealed that these cells express a variety of proteins (such as NF subunits) that are typically found in neural cells (4). The transformation of cells with Ad5, including the early region 1 (E1), has generated several human embryonic retinal cell lines (29), suggesting that Ad5 E1 may preferentially transform human neural lineages. Previous research has demonstrated that the efficient transformation of primary human amniocytes with the E1 gene of human Ad5 yielded stable cell lines which exhibited the morphological features of epithelial cells (30); a thorough immunocytochemical analysis confirmed the expression of epithelial cell markers and the analysis also revealed the expression of neuronal and glial marker proteins, such as nestin, vimentin, A2B5 and glial fibrillary acidic protein (GFAP) (30). In agreement with previous studies on 293 cells, these results suggest that epithelial and neuronal marker proteins are co-expressed in E1-transformed human amniotic fluid-derived cells. 293 cells exhibit chromosomal abnormalities, containing less than three times the number of chromosomes of a normal diploid human cell (31). Structural genomic alterations produced during cultivation for decades in different laboratories have been proposed to underlie the sometimes different conclusions drawn from experimentation with 293 cell lines (31). Thus, these differences may be the reason why we obtained the data demonstrating that 293 cells have characteristics of epithelial lineage cells.

In the present study, we examined the expression of epithelial marker proteins in 293 cells. Moreover, epithelial features were also investigated in non-transformed PKE cells, as well as in MDCK cells as a control cell population. Our data revealed that the 293 and PKE cells homogeneously expressed the neural cell marker, $\mathrm{N}$-cadherin. These cells were also found to express various epithelial marker proteins, cytokeratins 5/8, ZO-1, occludin and desmoglein 2 . Strictly speaking, desmoglein 2 is 
not a specific marker of epithelial cells, as it is expressed not only in epithelial cells, but also in various non-epithelial cells, such as myocardiac and Purkinje fiber cells of the heart (32). However, desmoglein 2 is found in all cell types that possess desmosomes and is the only desmoglein detected in diverse tissues, such as simple and transitional epithelia (33). Non-epithelial cell lines, including human fibroblast, rhabdomyosarcoma and glioma cell lines, do not express desmoglein 2. To the best of our knowledge, there is no evidence at present to suggest that neural cells express desmoglein 2 . As demonstrated by the present study for the first time to the best of our knowledge, the epithelioid characteristics of 293 cells were confirmed by the homogenous expression of the epithelial markers, cytokeratins $5 / 8$, as well as by the expression of the epithelial-specific contact proteins, ZO-1 (33) and occludin (10). Although endothelial cells express ZO-1 and occludin, they do not express E-cadherin, which is detected in 293 cells.

In the assembly of epithelia, surface interactions between adhesion molecules of the cadherin superfamily nucleate a cascade of protein-protein interactions that leads to the formation of additional junctions including desmosomes and tight junctions (34). It is generally accepted that before the extracellular domains of cadherins are capable of mediating adhesion, the cytoplasmic domains must first bind to catenins inside the cell $(16,35)$. The formation of this molecular complex confers adhesive strength by linking cadherins to the actin cytoskeleton and by clustering cadherin molecules, thus increasing the avidity of their interactions. We have previously demonstrated that an E-cadherin-expressing human colon carcinoma cell line lacking $\alpha$-catenin expression failed to organize desmosomes, and that the expression of $\alpha$-catenin in these cells by transfection resulted in the reorganization of desmosomes (36). Thus, the formation of desmosomes is dependent on the integrity of E-cadherin-catenin complexes.

$\mathrm{N}$-cadherin has been shown to rescue some, but not all, functionalities of E-cadherin during selective embryogenic events (37); gene replacement experiments have revealed that the strength of cellular adhesion provided by N-cadherin is sufficient to mediate morula compaction; however, it is insufficient for the subsequent formation of a fully polarized functional trophectoderm. A previous study also demonstrated that the first desmosomes in mouse embryos are formed between trophectoderm cells in early cavitating blastocysts (38), and, therefore, we conjectured that $\mathrm{N}$-cadherin may not act as a substitute for E-cadherin during desmosome formation. However, the attempted rescue by the exogenous expression of E-cadherin in 293 cells, failed to induce desmosome formation. Thus, it is clear that other factors are required for induction. In conclusion, the findings of the present study have established that 293 cells, by means of their epithelioid properties described herein, are suitable for use in studies on desmosome formation.

\section{Acknowledgements}

This study was supported by grants from the Ministry of Education, Culture, Sports, Science and Technology of Japan and a grant from Kodama Memorial Found for Medical Research. We wish to thank the Joint Research Laboratory at Kagoshima University Graduate School of Medical and Dental Sciences for the use of their facilities.

\section{References}

1. Graham FL, Smiley J, Russell WC and Nairn R: Characteristics of a human cell line transformed by DNA from human adenovirus type 5. J Gen Virol 36: 59-74, 1977.

2. Louis N, Evelegh C and Graham FL: Cloning and sequencing of the cellular-viral junctions from the human adenovirus type 5 transformed 293 cell line. Virology 233: 423-429, 1997.

3. Suzuki R, Sakamoto H, Yasukawa H, Masuhara M, Wakioka T, Sasaki A, Yuge K, Komiya S, Inoue A and Yoshimura A: CIS3 and JAB have different regulatory roles in interleukin- 6 mediated differentiation and STAT3 activation in M1 leukemia cells. Oncogene 17: 2271-2278, 1998.

4. Shaw G, Morse S, Ararat M and Graham FL: Preferential transformation of human neuronal cells by human adenoviruses and the origin of HEK 293 cells. FASEB J 16: 869-871, 2002.

5. Franke WW, Grund C and Achtstätter T: Co-expression of cytokeratins and neurofilament proteins in a permanent cell line: cultured rat PC12 cells combine neuronal and epithelial features. J Cell Biol 103: 1933-1943, 1986.

6. Damjanov I, Clark RK and Andrews PW: Cytoskeleton of human embryonal carcinoma cells. Cell Differ 15: 133-139, 1984.

7. Lee VM and Andrews PW: Differentiation of NTERA-2 clonal human embryonal carcinoma cells into neurons involves the induction of all three neurofilament proteins. J Neurosci 6: 514-521, 1986.

8. Schmidt U, Müller U, Metz KA and Leder LD: Cytokeratin and neurofilament protein staining in Merkel cell carcinoma of the small cell type and small cell carcinoma of the lung. Am J Dermatopathol 20: 346-351, 1998.

9. Farquhar MG and Palade GE: Junctional complexes in various epithelia. J Cell Biol 17: 375-412, 1963.

10. Furuse M, Hirase T, Itoh M, Nagafuchi A, Yonemura S, Tsukita S and Tsukita S: Occludin: a novel integral membrane protein localizing at tight junctions. J Cell Biol 123: 1777-1788, 1993.

11. Furuse M, Fujita K, Hiiragi T, Fujimoto K and Tsukita S: Claudin-1 and -2: novel integral membrane proteins localizing at tight junctions with no sequence similarity to occludin. J Cell Biol 141: 1539-1550, 1998.

12. Furuse M, Itoh M, Hirase T, Nagafuchi A, Yonemura S, Tsukita S and Tsukita S: Direct association of occludin with ZO-1 and its possible involvement in the localization of occludin at tight junctions. J Cell Biol 127: 1617-1626, 1994.

13. Itoh M, Furuse M, Morita K, Kubota K, Saitou M and Tsukita S: Direct binding of three tight junction-associated MAGUKs, $\mathrm{ZO}-1, \mathrm{ZO}-2$, and $\mathrm{ZO}-3$, with the $\mathrm{COOH}$ termini of claudins. J Cell Biol 147: 1351-1363, 1999.

14. Boller K, Vestweber D and Kemler R: Cell-adhesion molecule uvomorulin is localized in the intermediate junctions of adult intestinal epithelial cells. J Cell Biol 100: 327-332, 1985.

15. Ozawa M, Baribault $\mathrm{H}$ and Kemler R: The cytoplasmic domain of the cell adhesion molecule uvomorulin associates with three independent proteins structurally related in different species. EMBO J 8: 1711-1717, 1989.

16. Ozawa M, Ringwald M and Kemler R: Uvomorulin-catenin complex formation is regulated by a specific domain in the cytoplasmic region of the cell adhesion molecule. Proc Natl Acad Sci USA 87: 4246-4250, 1990.

17. Aberle H, Butz S, Stappert J, Weissig H, Kemler R and Hoschuetzky H: Assembly of the cadherin-catenin complex in vitro with recombinant proteins. J Cell Sci 107: 3655-3663, 1994.

18. Jou TS, Stewart DB, Stappert J, Nelson WJ and Marrs JA: Genetic and biochemical dissection of protein linkages in the cadherincatenin complex. Proc Natl Acad Sci USA 92: 5067-5071, 1995.

19. Buxton RS and Magee AI: Structure and interactions of desmosomal and other cadherins. Semin Cell Biol 3: 157-167, 1992.

20. Garrod DR, Merritt AJ and Nie Z: Desmosomal cadherins. Curr Opin Cell Biol 14: 537-545, 2002.

21. Nakanishi Y, Ogawa K, Yanagita K and Yamauchi C: Body measurement and some characteristics of inbred Clawn miniature pigs. Jpn J Swine Sci 28: 211-218, 1991.

22. Ozawa M: p120-independent modulation of E-cadherin adhesion activity by the membrane-proximal region of the cytoplasmic domain. J Biol Chem 278: 46014-46020, 2003.

23. Miyashita Y and Ozawa M: A dileucine motif in its cytoplasmic domain directs beta-catenin-uncoupled E-cadherin to the lysosome. J Cell Sci 120: 4395-4406, 2007.

24. Ozawa $M$ and Kobayashi W: Cadherin cytoplasmic domains inhibit the cell surface localization of endogenous E-cadherin, blocking desmosome and tight junction formation and inducing cell dissociation. PLoS One 9: e105313, 2014. 
25. Kalluri R and Weinberg RA: The basics of epithelial-mesenchymal transition. J Clin Invest 119: 1420-1428, 2009.

26. Ohkubo $\mathrm{T}$ and Ozawa M: The transcription factor Snail downregulates the tight junction components independently of E-cadherin downregulation. J Cell Sci 117: 1675-1685, 2004.

27. Stewart DB, Barth AI and Nelson WJ: Differential regulation of endogenous cadherin expression in Madin-Darby canine kidney cells by cell-cell adhesion and activation of beta-catenin signaling. J Biol Chem 275: 20707-20716, 2000.

28. Frank M, Ebert M, Shan W, Phillips GR, Arndt K, Colman DR and Kemler R: Differential expression of individual gammaprotocadherins during mouse brain development. Mol Cell Neurosci 29: 603-616, 2005.

29. Fallaux FJ and Hoeben RC: Safety of recombinant adenoviruses produced on adenovirus-transformed human cells. Dev Biol (Basel) 106: 489-497, 501-511, 2001.

30. Arnhold S, Post C, Glüer S, Hoopmann M, Wenisch S, Volpers C and Addicks K: Neuronal characteristics of amniotic fluid derived cells after adenoviral transformation. Cell Biol Int 32: 1559-1566, 2008.

31. Lin YC, Boone M, Meuris L, Lemmens I, Van Roy N, Soete A, Reumers J, Moisse M, Plaisance S, Drmanac R, et al: Genome dynamics of the human embryonic kidney 293 lineage in response to cell biology manipulations. Nat Commun 5: 4767, 2014.

32. Schäfer S, Koch PJ and Franke WW: Identification of the ubiq uitous human desmoglein, Dsg2, and the expression catalogue of the desmoglein subfamily of desmosomal cadherins. Exp Cell Res 211: 391-399, 1994.
33. Stevenson BR, Siliciano JD, Mooseker MS and Goodenough DA: Identification of ZO-1: a high molecular weight polypeptide associated with the tight junction (zonula occludens) in a variety of epithelia. J Cell Biol 103: 755-766, 1986.

34. Gumbiner B, Stevenson B and Grimaldi A: The role of the cell adhesion molecule uvomorulin in the formation and maintenance of the epithelial junctional complex. J Cell Biol 107: 1575-1587, 1988.

35. van Hengel J, Gohon L, Bruyneel E, Vermeulen S, Cornelissen M, Mareel M and von Roy F: Protein kinase C activation upregulates intercellular adhesion of alpha-catenin-negative human colon cancer cell variants via induction of desmosomes. J Cell Biol 137: 1103-1116, 1997.

36. Taniguchi T, Miyazaki M, Miyashita Y, Arima T and Ozawa M: Identification of regions of alpha-catenin required for desmosome organization in epithelial cells. Int J Mol Med 16: 1003-1008, 2005.

37. Kan NG, Stemmler MP, Junghans D, Kanzler B, de Vries WN, Dominis $\mathrm{M}$ and Kemler R: Gene replacement reveals a specific role for E-cadherin in the formation of a functional trophectoderm. Development 134: 31-41, 2007.

38. Fleming TP, Garrod DR and Elsmore AJ: Desmosome biogenesis in the mouse preimplantation embryo. Development 112: $527-539,1991$. 